\title{
REMARKS RELEVANT TO CLASSICAL MAXIMUM PRINCIPLES
}

CRISTIAN-PAUL DANET

Received 18 June 2003 and in revised form 4 August 2004

We extend, sharpen, or give independent proofs of classical maximum principles. We also concentrate on maximum principles for equations of higher order. All proofs (except for one) are derived via comparison principles. The two parts maybe read independently, but the whole paper is not self-contained.

\section{Introduction}

The purpose of this paper is to derive general estimates in the maximum norm for solutions of elliptic and parabolic equations, using some global-type comparison results. Our method has some attractive features, being elementary and applicable for a class of linear and nonlinear equations of second and higher order defined on nonsmooth domains. This idea was used for second-order equations and has proved to be a powerful tool.

Section 2.1 is devoted to maximum principles for second-order equations. First, we sharpen the classical bound for elliptic equations. Further, we study quasilinear equations and extend some results from the celebrated monograph [6, Problem 10.1, page 277] or reprove by different means some weaker variants of results in [6, Theorem 10.5 , page 266]. Then we consider parabolic equations and claim that stronger results (decay estimates) can be proved.

In Section 2.2, we transfer the same idea to the higher-order case. We will prove similar estimates in terms of boundary values of $\Delta^{j} u, 0 \leq j \leq m / 2-1$, where $m$ is the order of the elliptic equation.

A word on notations. The real function spaces and the definitions we use are all familiar, and are omitted (see, for details, [6]). But we note that L denotes a linear operator of the form

$$
\mathrm{L} u=a^{i j}(x) D_{i j} u+b^{i}(x) D_{i} u+c(x) u
$$

and Q denotes a quasilinear operator of the form

$$
\mathrm{Q} u=a^{i j}(x, u, D u) D_{i j} u+b(x, u, D u), \quad a^{i j}=a^{j i},
$$


where $x=\left(x_{1}, \ldots, x_{n}\right)$ is contained in a bounded domain $\Omega$ of $\mathbb{R}^{n}, n \geq 1$. The subscript $p$ indicates that we are concerned with parabolic operators, that is,

$$
\mathrm{L}_{p} u=-\frac{\partial u}{\partial t}+a^{i j}(x, t) D_{i j} u+b^{i}(x, t) D_{i} u+c(x, t) u
$$

where $(x, t) \in \Omega \times(0, T]=\Omega_{T}$. For elliptic quasilinear operators we will let $A$ denote the coefficient matrix $A=\left[a^{i j}(x, u, D u)\right]$ and set $\mathscr{D}^{*}=\sqrt[n]{\mathscr{D}}$, where $\mathscr{D}$ is the determinant of $A$.

\section{Results}

2.1. Maximum principles for second-order equations. The starting point is a slightly sharper version of [6, Theorem 3.7].

Theorem 2.1. Let $u \in C^{2}(\Omega) \cap C^{0}(\bar{\Omega})$ satisfy $\mathrm{L} u \geq f(=f)$ in $\Omega$, where $\mathrm{L}$ is elliptic, $b^{i}$, $i=1,2, \ldots, n$ are bounded, and $c \leq 0$. Assume also that $\Omega$ is contained in the strip between two planes of distance $d$. Then

$$
\sup _{\Omega} u(|u|) \leq \sup _{\partial \Omega} u^{+}(|u|)+C^{*} \sup _{\Omega} \frac{\left|f^{-}\right|}{\lambda}\left(\sup _{\Omega} \frac{|f|}{\lambda}\right) .
$$

Here $C^{*}=e^{(\beta+1) d} /(\beta+1), \beta=\sup _{\Omega}|b| / \lambda$, and $\lambda$ is the ellipticity constant.

Proof. Imitate [6, Proof of Theorem 3.7] with

$$
v(x)=\frac{e^{\eta d}}{\eta}\left(1-e^{-\eta x_{1}}\right) \sup _{\Omega} \frac{\left|f^{-}\right|}{\lambda}+\sup _{\partial \Omega} u^{+},
$$

where $\eta=1+\beta$.

Comments. (1) Theorem 2.1 is exactly the result of [6, Theorem 3.7] with $C=e^{(\beta+1) d}-1$ replaced by $C^{*}=e^{(\beta+1) d} /(\beta+1)$. Of course, if $\operatorname{diam}(\Omega) \geq 1$ and $\beta \geq 1 / 2$, we have $C^{*} \leq C$.

(2) In certain cases it is possible to relax the condition $c \leq 0$ (see [6, Corollary 3.8]). If parabolic operators of the form $\mathrm{L}_{p}$ are involved, then we can obtain similar estimates to (2.1) (for arbitrary $c$ ). A sharper (since we have only an integral norm of $f$ on the righthand side) form of estimate (2.1) is the Alexandrov-Bakelman maximum principle. The proof maybe found in [6, page 220].

We now pass to the quasilinear case. In the following, we are interested in proving a one-dimensional version of [6, Theorem 10.3] using a different method.

Theorem 2.2. Let $u \in C^{1}(\bar{\Omega}) \cap C^{2}(\Omega)$ be a solution of the equation

$$
a\left(x, u, u^{\prime}\right) u^{\prime \prime}+b\left(x, u, u^{\prime}\right)=0 \quad \text { in } \Omega=(0,1)
$$

and suppose there exist nonnegative constants $\mu_{1}$ and $\mu_{2}$ such that

$$
\frac{|b(x, z, p)|}{a(x, z, p)} \leq \mu_{1}|p|+\mu_{2}
$$

where $\mu_{1}<\pi$. 
Then

$$
\sup _{\Omega}|u| \leq \beta+C\left(\mu_{1}\right)\left[\pi(\beta-\alpha)+\mu_{2}\right]
$$

Here $u(0)=\alpha, u(1)=\beta$, and $\alpha<\beta$.

Proof. Setting $u(x)=y(x)+(\beta-\alpha) x+\alpha=y(x)+\varphi(x)$, we see that $y$ satisfies

$$
a\left(x, y+\varphi, y^{\prime}+\beta-\alpha\right) y^{\prime \prime}+b\left(x, y+\varphi, y^{\prime}+\beta-\alpha\right)=0 \quad \text { in } \Omega,
$$

and $y(0)=0, y(1)=0$.

By virtue of inequality $|y(x)| \leq \int_{0}^{x}\left|y^{\prime}\right| \leq\left(\int_{0}^{1}\left(y^{\prime}\right)^{2}\right)^{1 / 2}$, we need only estimate $\left\|y^{\prime}\right\|_{L^{2}(0,1)}$. We note that $\int_{0}^{1}\left(y^{\prime}\right)^{2}=-\int_{0}^{1}\left(y y^{\prime \prime}\right)$.

Hence using (2.6) we obtain

$$
\int_{0}^{1}\left(y^{\prime}\right)^{2} \leq \int_{0}^{1}|y| \frac{\left|b\left(x, y+\varphi, y^{\prime}+\beta-\alpha\right)\right|}{a\left(x, y+\varphi, y^{\prime}+\beta-\alpha\right)} \leq \mu_{1} \int_{0}^{1}|y|\left|y^{\prime}\right|+\left[\mu_{1}(\beta-\alpha)+\mu_{2}\right] \int_{0}^{1}|y| .
$$

Using Wirtinger's inequality

$$
\|y\|_{L^{2}(0,1)}^{2} \leq \frac{1}{\pi^{2}}\left\|y^{\prime}\right\|_{L^{2}(0,1)}^{2}
$$

which is valid for functions $y \in C^{1}[0,1]$ such that $y(0)=y(1)=0$, Cauchy's inequality with $\varepsilon$,

$$
t_{1} t_{2} \leq \frac{\varepsilon}{2} t_{1}^{2}+\frac{1}{2 \varepsilon} t_{2}^{2}
$$

and Holder's inequality, we get

$$
\begin{aligned}
& \int_{0}^{1} \mu_{1}|y|\left|y^{\prime}\right| \leq \mu_{1}\left(\int_{0}^{1}(y)^{2}\right)^{1 / 2}\left(\int_{0}^{1}\left(y^{\prime}\right)^{2}\right)^{1 / 2} \leq \mu_{1} \frac{1}{\pi} \int_{0}^{1}\left(y^{\prime}\right)^{2}, \\
& \int_{0}^{1}\left[\mu_{1}(\beta-\alpha)+\mu_{2}\right]|y| \leq \frac{\varepsilon}{2 \pi^{2}} \int_{0}^{1}\left(y^{\prime}\right)^{2}+\frac{1}{2 \varepsilon}\left[\mu_{1}(\beta-\alpha)+\mu_{2}\right]^{2} .
\end{aligned}
$$

Consequently,

$$
\left(1-\frac{\mu_{1}}{\pi}-\frac{\varepsilon}{2 \pi^{2}}\right) \int_{0}^{1}\left(y^{\prime}\right)^{2} \leq \frac{1}{2 \varepsilon}\left[\mu_{1}(\beta-\alpha)+\mu_{2}\right]^{2} .
$$

We take $\varepsilon>0$ small so that the term in brackets remains positive to obtain

$$
\sup _{\Omega}|y| \leq C\left(\mu_{1}\right)\left[\mu_{1}(\beta-\alpha)+\mu_{2}\right] .
$$

Replacing $y$ by $u-\varphi$, we obtain the desired estimate. 
52 Remarks relevant to classical maximum principles

Remark 2.1. If $\Omega=(a, b)$ is an arbitrary interval of finite length, then $u$ can also be estimated in terms of $u(a), u(b), \mu_{1}, \mu_{2}$, and $u^{\prime}(b)$. We do not give the proof here.

We note that we may reprove [6, Theorem 10.3] by choosing as comparison function

$$
w(x)=\sup _{\partial \Omega} u^{+}+\mu_{2} \frac{e^{\eta d}}{\eta}\left(1-e^{-\eta x_{1}}\right), \quad \eta=\mu_{1}+1,
$$

instead of $v$ (see the proof of [6, Theorem 10.3]).

One could prove a parabolic version of [6, Theorem 10.3]. Moreover, if $u$ is a classical solution of the problem

$$
\begin{gathered}
\mathrm{Q}_{p} u=0 \quad \text { in } \Omega \times(0, \infty), \\
u(x, 0)=\psi(x) \quad \text { in } \Omega, \\
u(x, t)=0 \quad \text { on } \partial \Omega \times(0, \infty),
\end{gathered}
$$

where $\mathrm{Q}_{p}$ is parabolic in $\Omega \times(0, \infty)$ and $b$ satisfies

$$
\frac{(\operatorname{sign} z) b(x, t, z, p)}{\varepsilon(x, t, z, p)} \leq \frac{\mu_{1}}{\|p\|} \quad \forall(x, t, z, p) \in \Omega \times \mathbb{R} \times \mathbb{R} \times \mathbb{R}^{n}
$$

$\left(\mu_{1}>0\right.$ is a constant and $\left.\varepsilon(x, t, z, p)=a^{i j}(x, t, z, p) p_{i} p_{j}\right)$ then the solution has the following decay property:

$$
|u(x, t)| \leq e^{-\alpha t}, \quad x \in \Omega, t>0 .
$$

Here $\alpha$ is a positive constant.

The proof is similar to that of [3, Lemma 3] and is left to the reader.

A very general maximum principle is stated in [6, Theorem 10.5]. It tells us that if $u$ solves $\mathrm{Q} u \geq 0$ in $\Omega$ and if there exist nonnegative functions $g \in \mathrm{L}_{\text {loc }}^{n}\left(\mathbb{R}^{n}\right), h \in \mathrm{L}^{n}(\Omega)$ such that

$$
\begin{gathered}
\frac{(\operatorname{sign} z) b(x, z, p)}{n D^{*}} \leq \frac{h(x)}{g(p)} \quad \forall(x, z, p) \in \Omega \times \mathbb{R} \times \mathbb{R}^{n}, \\
\int_{\Omega} h^{n} d x<\int_{\mathbb{R}^{n}} g^{n} d p,
\end{gathered}
$$

then a maximum principle is valid.

Our aim is to show that under strong conditions on the coefficients $a^{i j}$ and on $h$ the maximum principle holds even if $g \notin \mathrm{L}_{\text {loc }}^{n}\left(\mathbb{R}^{n}\right)$. 
Theorem 2.3. Let $u \in C^{0}(\bar{\Omega}) \cap W_{\text {loc }}^{2, n}(\Omega)$ satisfy $\mathrm{Q} u \geq 0(=0)$ in $\Omega$. Suppose that $b$ satisfies the structure condition (2.17) with $h$ bounded in $\Omega$ and $g(p)=\|p\|^{-k}, k>1$. If in addition $\mathrm{Q}$ is elliptic with $a^{i j}(x, z, p) \geq 0$ in $\Omega \times \mathbb{R} \times \mathbb{R}^{n}$, for $i \neq j$, then the estimate

$$
\sup _{\Omega} u(|u|) \leq 1+\sup _{\partial \Omega} u^{+}(|u|)
$$

holds.

Proof. Suppose that $\Omega$ lies in the cube

$$
K=\left\{x \in \mathbb{R}^{n} \mid 0<x_{i}<d, i=1,2, \ldots, n\right\}
$$

where $d=\operatorname{diam}(\Omega)$.

We consider the function

$$
w(x)=\sup _{\partial \Omega} u^{+}+1-\frac{e^{-\eta\left(x_{1}+\cdots+x_{n}\right)}}{\eta},
$$

where the constant $\eta>1$ is to be chosen later.

Let $u \in C^{0}(\bar{\Omega}) \cap W_{\text {loc }}^{2, n}(\Omega)$ satisfy $\mathrm{Q} u \geq 0$ in $\Omega$ and define $\overline{\mathrm{Q}}$ by

$$
\overline{\mathrm{Q}} w=a^{i j}(x, u, D w) D_{i j} w+b(x, u, D w) .
$$

It is then not difficult to see that

$$
\begin{aligned}
\overline{\mathrm{Q}} w & =-\eta e^{-\eta\left(x_{1}+\cdots+x_{n}\right)} \cdot \sum_{i, j} a^{i j}(x, u, D w)+b(x, u, D w) \\
& \leq-\eta e^{-\eta\left(x_{1}+\cdots+x_{n}\right)} \cdot \sum_{i} a^{i i}(x, u, D w)+n \mathscr{D}^{*}|h(x)| e^{-\eta\left(x_{1}+\cdots+x_{n}\right)}
\end{aligned}
$$

in $\Omega^{+}=\{x \in \Omega \mid u(x)>0\}$.

But

$$
\operatorname{det} A \leq\left(\frac{\operatorname{trace} A}{n}\right)^{n}
$$

Since $h$ is bounded in $\Omega$ we can choose $M$ such that $|h| \leq M$ in $\Omega$ to obtain

$$
\overline{\mathrm{Q}} w \leq e^{-\eta\left(x_{1}+\cdots+x_{n}\right)} \cdot \sum_{i} a^{i i}(x, u, D w)(-\eta+M) \quad \text { in } \Omega^{+} .
$$

Setting $\eta=M+1$ we have

$$
\overline{\mathrm{Q}} w<0 \leq \overline{\mathrm{Q}} u \text { in } \Omega^{+},
$$

and hence (2.19) follows from [6, Theorem 10.1]. 


\section{Remarks relevant to classical maximum principles}

Remark 2.2. The hypothesis that $a^{i j}(x, z, p) \geq 0$ in $\Omega \times \mathbb{R} \times \mathbb{R}^{n}$ can be replaced by $a^{i j}$ bounded in $\Omega \times \mathbb{R} \times \mathbb{R}^{n}(i \neq j)$.

The following result provides an extension of the maximum principle [6, (10.37), page 277].

Theorem 2.4. Let $u \in C^{2}(\Omega) \cap C^{0}(\bar{\Omega})$ satisfy $\mathrm{Q} u \geq 0(=0)$ in $\Omega$. Suppose the following.

(i) $a^{i j}(x, z, 0) \xi_{i} \xi_{j} \geq 0$ for all $\xi \in \mathbb{R}^{n},(x, z) \in \Omega \times \mathbb{R}$ and

$$
z \cdot b(x, z, 0) \leq 0
$$

for all $x \in \Omega,|z| \geq M$ (here $M$ is a positive constant).

Then

$$
\sup _{\Omega} u(|u|) \leq \max \left\{\sup _{\partial \Omega} u^{+}(|u|), M\right\}
$$

(ii) $\Omega$ lies between two parallel planes a distance 1 apart, $a^{i j}=\delta^{i j}$, and there exists $a$ constant $M>0$ such that

$$
z \cdot b(x, z, p) \leq z^{2}+\mu_{1} \quad \forall x \in \Omega,|z|>M, p \in \mathbb{R}^{n},
$$

where $\mu_{1} \geq 0$. If in addition there exists a constant $L_{1}>0$ such that

$$
\left|b(x, z, p)-b\left(x, z_{1}, p_{1}\right)\right| \leq L_{1}\left|p-p_{1}\right| \quad \forall x \in \Omega, z, z_{1} \in \mathbb{R}, p, p_{1} \in \mathbb{R}^{n},
$$

then

$$
\sup _{\Omega} u(|u|) \leq \sup _{\partial \Omega} u^{+}(|u|)+C,
$$

where $C=C\left(\mu_{1}, M\right)$.

(iii) $Q$ is strictly elliptic in $\Omega$, and b satisfies (2.30). Also suppose that for some $k \in$ $\{1,2, \ldots, n\}$, there exists a constant $L_{2}>0$ such that

$$
\left|a^{k k}(x, z, p)-a^{k k}\left(x, z_{1}, p_{1}\right)\right| \leq L_{2} \cdot\left|p-p_{1}\right|
$$

for all $x \in \Omega, z, z_{1} \in \mathbb{R}, p, p_{1} \in \mathbb{R}^{n}$. If

$$
z \cdot b(x, z, p) \leq \mu_{1} \cdot|z|^{\alpha} \quad \forall x \in \Omega, z \in \mathbb{R}, p \in \mathbb{R}^{n},
$$

where $\mu_{1}>0, \alpha \geq 3$, then $\sup _{\Omega} u(|u|) \leq \sup _{\partial \Omega} u^{+}(|u|)+C$ with $C=C\left(\alpha, \operatorname{diam}(\Omega), \lambda_{0}\right)$, where $\lambda_{0}$ is a lower bound for the minimum of eigenvalues of $\left[a^{i j}(x, z, p)\right],(x, z, p) \in \Omega \times$ $\mathbb{R} \times \mathbb{R}^{n}$.

Proof. (i) We define $\overline{\mathrm{Q}}$ as in the proof of Theorem 2.3; namely for $u \in C^{0}(\bar{\Omega}) \cap C^{2}(\Omega)$ that satisfies $\mathrm{Q} u \geq 0$ in $\Omega$, we set

$$
\overline{\mathrm{Q}} v=a^{i j}(x, u, D v) D_{i j} v+b(x, u, D v)
$$


By considering the function

$$
v(x)=\max \left\{\sup _{\partial \Omega} u^{+}, M\right\},
$$

we obtain

$$
\overline{\mathrm{Q}} v=b(x, u, 0) \leq 0 \quad \text { in } \Omega^{+} .
$$

Estimate (2.28) for $\sup _{\Omega} u$ follows by [11, Corollary III, page 306]. Replacing $u$ by $-u$, we obtain estimate (2.28) for $\sup _{\Omega}|u|$.

(ii) Assume that $\Omega$ is contained in the strip $\pi / 6 \sqrt{2}<\delta_{1}<x_{1}<\delta_{2}<5 \pi / 6 \sqrt{2}$, where $\delta_{2}-\delta_{1}=1$. We also assume initially that $u \leq 0$ on $\partial \Omega$, that is, $\sup _{\partial \Omega} u^{+}=0$.

A comparison function $v$ is defined by

$$
v(x)=2\left(\mu_{1}+1\right) \cdot(M+1) \cdot \sin \left(\sqrt{2} x_{1}\right) .
$$

We then get

$$
\mathrm{Q} v \leq \mathrm{Q} u \quad \text { in } \Omega,
$$

and the result with $\sup _{\partial \Omega} u^{+}=0$ follows from the refined form of [11, Corollary III, page 307]. By replacing $u$ with $u-\gamma$, where $\gamma=\sup _{\partial \Omega} u^{+}$we obtain estimate (2.31) for subsolutions.

(iii) As in the proof of (ii) we can assume initially that $u \leq 0$ on $\partial \Omega$, and that $\Omega$ lies in the strip $0<x_{1}<d, d=\operatorname{diam}(\Omega)$.

Defining the function $v$ as

$$
v(x)=r\left(e^{\eta d}-e^{\eta x_{1}}\right)
$$

where the positive constants $r, \eta$ will be chosen below, we see that

$$
\mathrm{Q} v=-r \eta^{2} e^{\eta x_{1}} a^{11}(x, v, D v)+b(x, v, D v) \quad \text { in } \Omega \text {. }
$$

By hypothesis $a^{11}(x, z, p) \geq \lambda_{0}$ in $\Omega \times \mathbb{R} \times \mathbb{R}^{n}$. Hence

$$
\mathrm{Q} v \leq r e^{\eta x_{1}}\left[-\lambda_{0} \eta^{2}+\mu_{1} r^{\alpha-2}\left(e^{\eta d}-1\right)^{\alpha-1}\right] \quad \text { in } \Omega .
$$

We choose $\eta=\left(\left(1+\mu_{1}\right) / \lambda_{0}\right)^{1 / 2}, r=1 /\left(e^{\eta d}\right)^{(\alpha-1) /(\alpha-2)}$, and obtain

$$
\mathrm{Q} v<0 \leq \mathrm{Q} u \text { in } \Omega \text {. }
$$

The proof may be effected by using an argument similar to that of (ii).

Comments. Since we have used a better comparison result, the maximum principle in [6, (10.37), page 277] becomes a particular case of our principle (2.28). A weaker form 
of this principle appears in [10]. The cases of Theorem 2.4(ii) and (iii) can be viewed as extensions of (10.37).

Conditions (2.30) and (2.32) in the hypothesis of Theorem 2.4(iii) can be replaced by the following:

(i) $b$ is strictly decreasing in $z$ for each fixed $(x, p) \in \Omega \times \mathbb{R}^{n}$,

(ii) for some $k, a^{k k}$ is increasing in $z$ for each fixed $(x, p) \in \Omega \times \mathbb{R}^{n}$.

A parabolic version of Theorem 2.4 maybe proved in a similar manner (using the wellknown Nagumo-Westphal lemma in [11, page 187] instead of Corollary III). However, this result is a particular case of [8, Theorem 2.9, page 23] with $\phi(s)=\alpha t^{\beta}$, where $\alpha>0$, $\beta \geq 1$. For some sharper results, that is, decay estimates, the reader is referred to [4].

2.2. Maximum principles for higher-order equations. Maximum principles for equations of higher order have been developed by various authors (see $[1,2,5,9,12])$ using different methods.

Our approach (based on comparison methods) differs considerably from those in the above quoted works. Unfortunately, by using this method we cannot strive to obtain maximum principles for a broad class of equations. However, it allows us to treat the subsolution case.

Theorem 2.5. Let $u \in W_{\text {loc }}^{4, n}(\Omega) \cap C^{2}(\bar{\Omega})$ satisfy $\mathrm{B} u \leq f(=f)$ in $\Omega$, where $\mathrm{B}$ is an elliptic operator given by $\mathrm{B} u=\mathrm{L}^{2} u-\eta \mathrm{L} u+\gamma u$, and where the constants $\eta>0$ and $\gamma$ satisfy $0 \leq$ $4 \gamma \leq \eta^{2}$, and $\mathrm{L} u=a^{i j} D_{i j} u$ (a $a^{i j}$-constants). Then

$$
\sup _{\Omega} u(|u|) \leq \sup _{\partial \Omega} u^{+}(|u|)+C_{1} \sup _{\partial \Omega} \frac{-(\mathrm{L} u)^{-}}{\lambda}\left(\frac{|\mathrm{L} u|}{\lambda}\right)+C_{2} \sup _{\partial \Omega} \frac{f^{+}}{\lambda^{2}}\left(\frac{|f|}{\lambda^{2}}\right),
$$

where $C_{1}, C_{2}$ are constants depending only on diameter of $\Omega$. Here $\lambda$ is the ellipticity constant for the operator $\mathrm{L}$.

Proof. Without loss of generality, we may assume that $\Omega$ lies in the strip $1<x_{1}<d+1$, where $d$ is the diameter of $\Omega$. We suppose first that $\mathrm{B} u \leq f$ in $\Omega$. Our strategy is to choose a comparison function $v$. We set

$$
\begin{aligned}
v(x)= & \sup _{\partial \Omega} u^{+}+\left(\frac{(d+1)^{2}}{2}-\frac{x_{1}^{2}}{2}\right) \sup _{\partial \Omega} \frac{-(\mathrm{L} u)^{-}}{\lambda} \\
& +\left[\frac{3 x_{1}^{2}}{4}+\left(\frac{(d+1)^{2}}{2} \log (1+d)-\frac{x_{1}^{2}}{2} \log x_{1}\right)\right] \cdot(d+1)^{2} \cdot \sup _{\Omega} \frac{f^{+}}{\lambda^{2}} .
\end{aligned}
$$

Obviously $u \leq v$ on $\partial \Omega$.

By ellipticity we have $a^{11} \geq \lambda$, and hence

$$
\mathrm{L} v=\frac{a^{11}}{\lambda}\left(\inf _{\partial \Omega}(\mathrm{L} u)^{-}-(d+1)^{2} \log x_{1} \sup _{\Omega} \frac{f^{+}}{\lambda}\right) \leq \inf _{\partial \Omega}(\mathrm{Lu})^{-} \leq \mathrm{Lu} \quad \text { on } \partial \Omega .
$$


Since

$$
\mathrm{B}(v-u) \geq\left(\frac{a^{11}}{\lambda}\right)^{2} \cdot \frac{(d+1)^{2}}{x_{1}^{2}} \cdot \sup _{\Omega} f^{+}-f \geq \sup _{\Omega} f^{+}-f \geq 0 \quad \text { in } \Omega,
$$

we obtain the result for functions $C^{2}(\bar{\Omega}) \cap C^{4}(\Omega)$ by an extension (we interchanged the symbols $\geq$ and $\leq$ and replaced $\Delta u$ by the elliptic operator $L u$ ) of [7, Theorem 2] (see also the remark of Goyal and Schaefer in [7], top of page 278). We note that the constants $a, b$ in [7, Theorem 2] are here $\eta$, respectively, $\gamma$. But [7, Theorem 2] remains valid for functions in $C^{2}(\bar{\Omega}) \cap W_{\text {loc }}^{4, n}(\Omega)$ (the proof is left to the reader) and hence the desired result follows.

The result for solutions is obtained by replacing $u$ with $-u$.

Comments. If $u$ is a solution of $\mathrm{B} u=f$ in $\Omega$, then Theorem 2.5 becomes a particular case of [12, Corollary 13]. We can use similar means to extend the result of Theorem 2.5 to subsolutions (solutions) of

$$
\mathrm{B}_{1} u=\Delta^{2} u-(c+d) \Delta u+c d u \leq f(=f) \quad \text { in } \Omega,
$$

where $c$ is a positive constant and $d$ is a positive function in $\Omega$ in the class $C^{0}(\Omega)$. We observe that this last result cannot be derived from results in $[1,2,5,9,12]$, even if $u$ is a solution of $\mathrm{B}_{1} u=f$ in $\Omega$.

It is worth noticing that it is also possible to extend Theorem 2.5 to operators of order $2 m$ and hence obtain corresponding estimates for solutions of

$$
\Delta^{m} u+c_{1} \Delta^{m-1} u+\cdots+(-1)^{m+1} c_{m} u=f \quad \text { in } \Omega \text {, }
$$

if the constants $c_{1}<0, c_{2}, c_{3}, \ldots, c_{m}>0$ are chosen appropriately.

We save a tree and leave this as an exercise for the reader.

\section{Acknowledgments}

This paper was written while the author was visiting the Technical University of Munich. The author is grateful to Professor A. M. Hinz for some discussions concerning this research. This work was supported by a KAAD Grant.

\section{References}

[1] S. Agmon, Maximum theorems for solutions of higher order elliptic equations, Bull. Amer. Math. Soc. 66 (1960), 77-80.

[2] D. R. Dunninger, Maximum principles for solutions of some fourth-order elliptic equations, J. Math. Anal. Appl. 37 (1972), 655-658.

[3] J. P. G. Ewer, On the asymptotic properties of a class of nonlinear parabolic equations, Appl. Anal. 13 (1982), no. 4, 249-260.

[4] J. P. G. Ewer and L. A. Peletier, On the asymptotic behavior of solutions of semilinear parabolic equations, SIAM J. Appl. Math. 28 (1975), 43-53.

[5] A. Friedman, B. Hu, and J. J. L. Velazquez, Asymptotics for the biharmonic equation near the tip of a crack, Indiana Univ. Math. J. 47 (1998), no. 4, 1327-1395. 
[6] D. Gilbarg and N. S. Trudinger, Elliptic Partial Differential Equations of Second Order, Classics in Mathematics, Springer-Verlag, Berlin, 2001.

[7] V. B. Goyal and P. W. Schaefer, Comparison principles for some fourth order elliptic problems, Ordinary and Partial Differential Equations (Dundee, Scotland, 1982), Lecture Notes in Mathematics, vol. 964, Springer-Verlag, New York, 1982, pp. 272-279.

[8] O. A. Ladyzenskaya, V. A. Solonikov, and N. N. Uralceva, Linear and Quasilinear Equations of Parabolic Type, Am. Math. Soc. Transl., vol. 23, American Mathematical Society, Rhode Island, 1968.

[9] P. W. Schaefer and W. Walter, On pointwise estimates for metaharmonic functions, J. Math. Anal. Appl. 69 (1979), no. 1, 171-179.

[10] J. Serrin, The problem of Dirichlet for quasilinear elliptic differential equations with many independent variables, Philos. Trans. Roy. Soc. London Ser. A 264 (1969), 413-496.

[11] W. Walter, Differential and Integral Inequalities, Ergebnisse der Mathematik und ihrer Grenzgebiete, vol. 55, Springer-Verlag, New York, 1970.

[12] C. P. Zhou, Maximum principles for elliptic systems, J. Math. Anal. Appl. 159 (1991), no. 2, 418-439.

Cristian-Paul Danet: Department of Applied Mathematics, University of Craiova, 200585 Craiova, Romania

E-mail address: cristiandanet@yahoo.com 


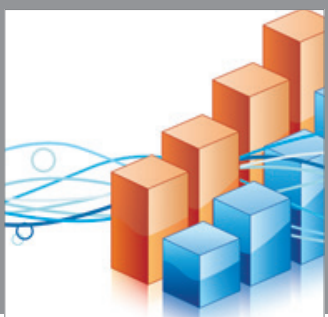

Advances in

Operations Research

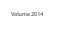

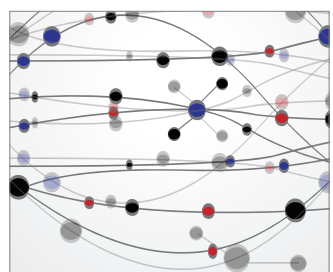

\section{The Scientific} World Journal
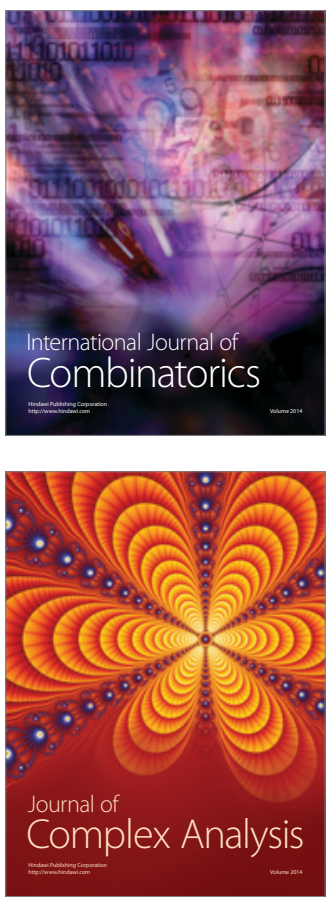

International Journal of

Mathematics and

Mathematical

Sciences
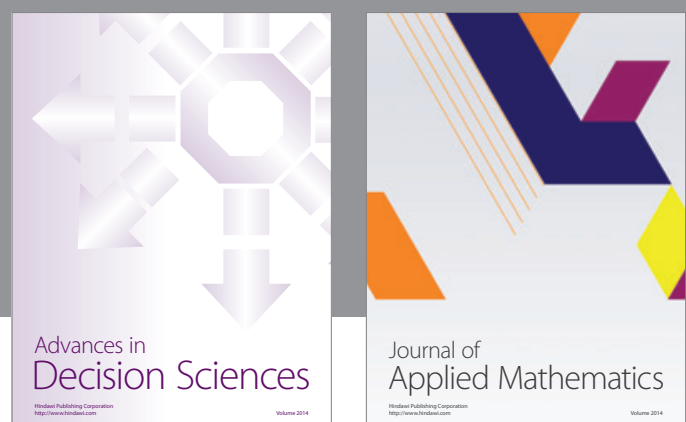

Journal of

Applied Mathematics
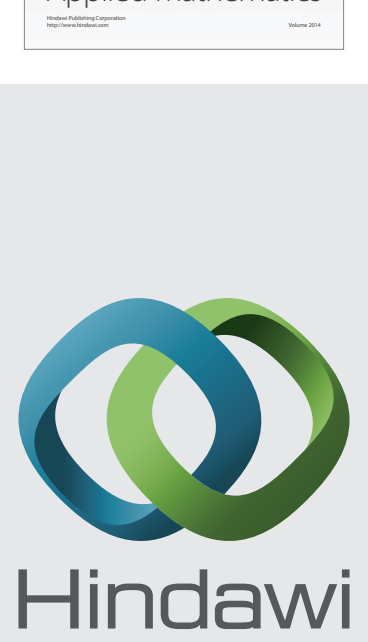

Submit your manuscripts at http://www.hindawi.com
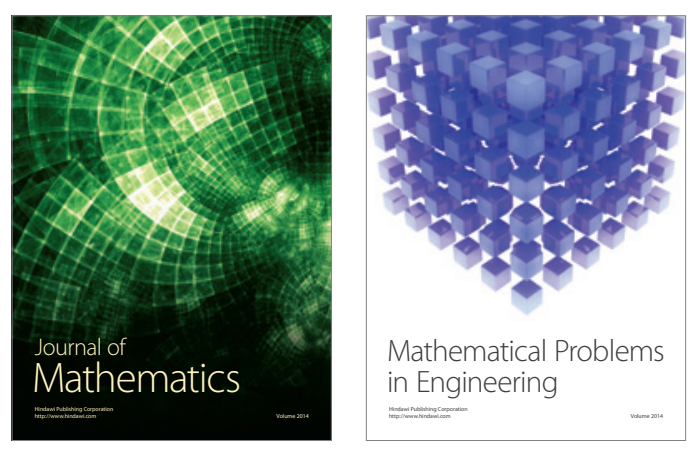

Mathematical Problems in Engineering
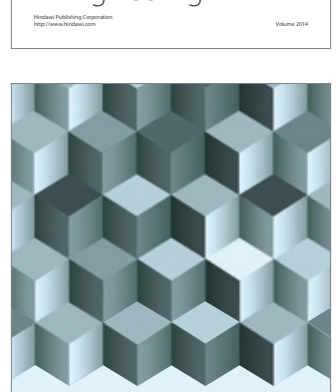

Journal of

Function Spaces
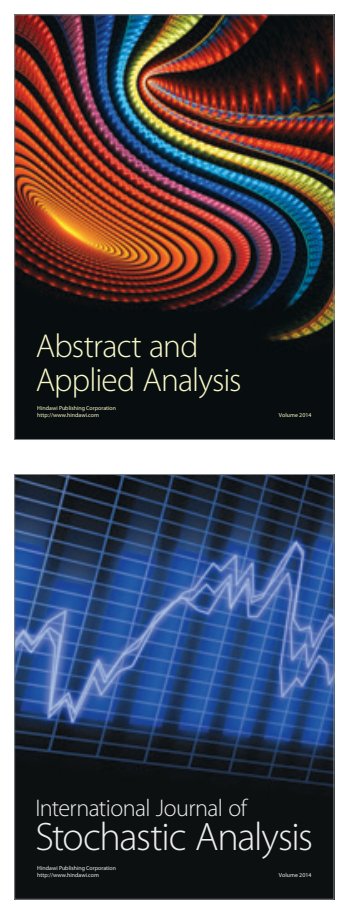

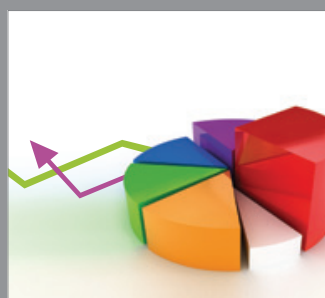

ournal of

Probability and Statistics

Promensencen
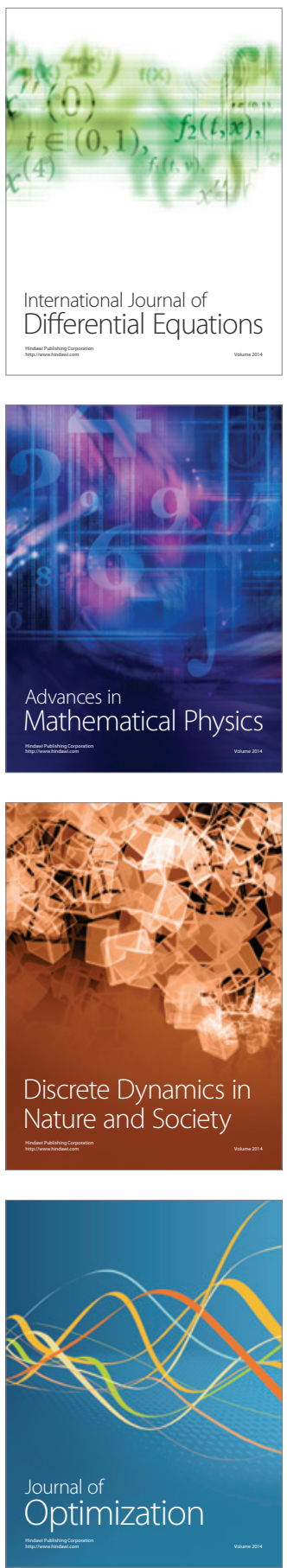